\title{
The Paradox of Strategic Management Accounting
}

\section{Bill Nixon}

Emeritus Professor, University of Dundee, 1 Perth Road, Dundee, DD1 4HN, United Kingdom, email: w.a.j.nixon@dundee.ac.uk

and

\section{John Burns * $\wedge$}

Professor of Management \& Accountancy, University of Exeter, Business School, Streatham Court, Rennes Drive, Exeter, EX4 3PU, United Kingdom, email: j.e.burns@exeter.ac.uk

\footnotetext{
* Corresponding author
}

^ John Burns is also (part-time) visiting Professor at WHU Otto Beisheim School of Management (Germany) and the Centre for Empirical Research on Organizational Control, Örebro University (Sweden)

\section{Acknowledgement}

The authors would like to thank the Chartered Institute of Management Accountants (CIMA) and the University of Exeter (Business School) for funding our research. 


\section{Abstract}

The evidence that strategic management accounting (SMA) techniques have not been adopted widely and that developments in the SMA literature seem to have languished may be consistent with the relatively short lifecycle of most strategic management (SM) tools and many concepts. Nevertheless, there is an inherent contradiction between the apparent decline of SMA and the sustained growth in the number of concepts, models, tools, theoretical perspectives, disciplines, academic and professional journals and consultancy practices that populate the SM domain.

This paradox of SMA is explored in the context of the evolution of the SM literature, SMA practice, as exemplified by two recent case studies, and the cognate literatures of management control, performance measurement and knowledge management. It transpires that the SMA literature is based in large part on a narrow, first-era, view of the SM literature that reached maturity with Michael Porter's industry analysis model and generic competitive strategies.

The second era of SM that began in 1977 with a move to a more internal, resource-based view of the firm and competitive advantage has been mostly neglected by the extant SMA literature. However, to judge from the small number of published case studies, SMA practices are developing in line with their strategy formulation and organisational processes. The links among the bundle of techniques that are usually included in SMA and between SMA and cognate literatures need to be integrated into a coherent, cohesive framework to complement SM. 


\section{Keywords}

Strategic Management Accounting, Strategic Management, New Product Design and

Development, Performance Measurement, Knowledge Management, Management Control, Resource-Based View, Strategy-as-Practice, Complementarities 


\section{The Paradox of Strategic Management Accounting}

\section{The test of time}

We all know that change has become the most predictable characteristic of the business and economic environments. What is more significant is that the rate of change is accelerating (Nadler and Tushman, 1999; Williamson, 1999; Jacobides, 2010; Grant and Jordan, 2012); this is the experience of the last 50 years (ref. Table 1). In every decade since the financial planning era of the 1950 s new external environmental challenges have shaped the formulation, implementation and control of strategy in organisations and the related research and literature (Bowman et al., 2002).

There are many definitions of the strategic management (SM) process but there is a broad consensus that key activities are (1) development of a grand strategy, purpose or sense of direction, (2) formulation of strategic goals and plans to achieve them, (3) implementation of plans, and (4) monitoring, evaluation and corrective action (Ward, 1992; Bartlett and Ghoshal, 1994; Kreitner, 2004; Grant and Jordan, 2012). The relative emphasis on each activity varies over time and among organisations. Of course, endogenous factors, such as corporate culture and the strategic orientation of senior management, also influence the SM process in organisations (Child, 1984; Porter, 2000); a separate influence on the SM literature and the research agenda are the views of particular authors and certain journal publications (Furrer et al., 2008). 
One salient feature of the evolution of SM is that 'strategic management does not lend itself to long-lasting techniques, methods or specific approaches - no particular tool or prescription has stood the test of time' (Cummings and Daellenbach, 2009, p. 256). This obsolescence reflects the need to constantly adapt and realign strategy and organisational arrangements to an increasingly turbulent and complex external business environment. The history of strategic management accounting (SMA) suggests that it may be another approach to SM that has not stood 'the test of time' (op. cit.). A comprehensive review of the SMA literature and the evidence of its use found that 'SMA or SMA techniques have not been adopted widely, nor is the term SMA widely understood or used' (Langfield-Smith, 2008, p. 204). There is a sharp contrast, a paradox, between the vibrant, eclectic development in the practice and theory of SM in the last 50 years, reflected in the substantive practice-oriented and academic literatures, and the corresponding developments in SMA that seem 'to have languished' (Seal, 2010, p. 107) and look more like a blip or a fad.

The apparent low adoption of SMA techniques also seems inconsistent with business operating environments that demand more information, including management accounting. Intensification of competition and new technologies, especially web-based technologies, are transforming business models, creating new businesses and wiping out others (Bughin et al., 2010; Arthur, 2011). The economic crisis that erupted in 2008 has intensified the need for careful financial, particularly cost and cash flow, management and added another dimension to the changing external environment of business (Hopwood, 2009).

The evident decline of interest in SMA since the 'glory decade' of the 1990s (Langfield-Smith, 2008, p. 208) also seems at odds with the long, steady development and expanding interest in cognate accounting areas intended to 
support SM, such as management control systems, performance measurement and knowledge management. Indeed, Kaplan and Norton claimed in 2001 that the Balanced Scorecard had transformed from 'performance measurement to strategic management' (Kaplan and Norton, 2001a, p. 87).

The discussion that follows addresses these three perspectives of SMA; there is, it seems, an urgent need for better integration of the SMA literature with the SM literature, with practice in its organisational and strategic contexts and with related accounting-based approaches that are also intended to support SM. The remainder of our paper is structured as follows. Section 2, below, provides a brief history of the evolution of SM in the last 50 years (see also Table 1) in order to consider how SMA relates to the practice issues, theories and trends in SM. Section 3 evaluates the scope of the SMA literature in the context of the evolution of SM. Section 4 reviews two recent case studies that exemplify the key role played by accounting in the SM of the new product design and development process. 'Confrontation strategy' (Cooper, 1995, p.9) is now much more complex and challenging than balancing 'the survival triplet' (ibid., p.ix) of 'cost, quality and functionality' (ibid.). Organisations must now pay greater attention to service, customer experience, customisation, brand identity and the environmental dimension of products and services. Section 5 , SMA and related literatures, is inspired, in part, by the emphasis on integration of processes, phases, participants and activities within the new product design and development function. Stronger links among the SMA and cognate literatures ought to help to provide a conceptual basis to strengthen the links between the analysis and synthesis of cost and management accounting data and between strategic objectives and operational targets. Finally, Section 6, our conclusion, considers how 
better alignment can be achieved between the practice and theories of SM and those of (management) accounting, in the context of past and likely future trends of SM,

\section{The SM literature}

\subsection{Seminal works}

The origins of SM can be traced to three works in particular: Chandler's 'Strategy and Structure', 1962; Ansoff's 'Corporate Strategy', 1965; and the textbook, 'Business Policy: Text and Cases', Learned et al., 1965 (Herrmann, 2005). Chandler (1962) defined strategy in terms of decisions about long-term goals and the resources and courses of action needed to attain those goals. Nevertheless, the focus of organisations, consultants and researchers in the 1960s and 70s was on planning more than on implementation. An indication of the emphasis on planning is the journal, Long Range Planning that was established in 1969 and which recently commemorated its $40^{\text {th }}$ year with a special issue (Cummings and Daellenbach, 2009). The 1960 s and 70 s also saw a move away from a deterministic, one-best-way approach to a more contingent perspective where organisations need to align or 'fit' resources to opportunities and threats in their external environment (Woodward, 1965; Lawrence and Lorsch, 1967).

\subsection{A new era: A shift to a resource-based view of the organisation}

Increasing volatility and unpredictability of the external environment in the 1970 s exposed the limitations of planning that took inadequate account of the 
organisational design and competencies required to adapt quickly to new threats and opportunities in order to attain strategic goals. As a result a stream of research began to focus on the processes by which explicit and implicit strategies evolved. Mintzberg, 1978, adduced the concept of 'emergent' strategy in contrast to the traditional, classical, 'deliberate' strategy that is the prerogative of top management. 'Realised' strategy, or actual performance achieved, according to Mintzberg, is attributable to both deliberate and emergent strategies (discussed below in section 2.7).

By the mid 1970s it was evident that the recurring failure of many organisations to achieve strategic goals could not be explained entirely by the weakness of the economies, especially those of the USA and Britain, in which they were operating. Researchers acknowledged the need for tighter links between strategy formulation and organisational design, for more analytical rigor, a better understanding of organisational resources and processes, and a need to both broaden the scope and strengthen the cohesion of the field.

Consequently, following a conference in Pittsburgh in 1977: 'researchers changed the name of the field from business policy to 'strategic management', to signal its move to a more empirically oriented discipline' (Herrmann, 2005, p. 115). The Strategic Management Journal, the premier journal in the field, was established in 1979 to signal a new approach to attainment of sustainable competitive advantage based on core competencies and resources, especially difficult-to-imitate intangibles, such as culture, intellectual property and capital, creativity, adaptability, reputation and relationships with stakeholders (Herrmann, 2005; Mele and Guillen Parra, 2006; Furrer et al., 2008). This move to a more internal, resource-based view of the 
organisation and competitive advantage can be seen as a turning point, a discontinuity that started the 'second era of ferment' in SM (Herrmann, 2005, p. 118).

The early 1980s was a key period in the evolution of both SM and SMA. The resource-based view was gaining acceptance among managers, consultants and researchers confronted with intensification of foreign competition and globalisation of markets. Meanwhile the established, first-era notion of competitive advantage that is primarily determined by external environmental factors reached a maturity with Porter's industry (Five Forces) analysis model and generic competitive strategies (1980, 1985). First-era SM was about aligning an organisation's strategy to a somewhat vague external environment; Porter's Five Forces framework provided a much more specific basis for systematic analysis of industry attractiveness. Not surprising, perhaps, Porter's framework focuses on the economic environment and neglects related environments, such as the technological, political, social and legal, that interact with economic components and activities and affect strategic outcomes.

Development of the SM literature was especially evident following the formal name change in 1977 and the establishment of Strategic Management Journal in 1979 to reflect the shift in emphasis from the strategic planning of business policy to the resource-based view of SM. A content analysis, undertaken by Furrer et al., 2008, comprising a review of 2,125 articles, published in the 26-year period 1980 2005, in four leading strategic management journals, namely Academy of Management Journal, Academy of Management Review, Administrative Science Quarterly and Strategic Management Journal, indicated that the evolution of SM in this period was almost linear insofar as all the keywords moved in the same direction. 
The internal focus of the resource-based view that began in the 1970s increased along with an interest in corporate-level strategy; conversely, decreased interest was observed in the notion of strategy as fit and in the role of top managers. The focus of the resource-based view on the internal organisational arrangements evolved to integrate elements of knowledge management and organisational economics, especially agency theory and transaction cost economics (Bowman et al., 2002; Furrer et al., 2008).

\subsection{Key issues in SM}

The content analysis of the 2,125 articles (Furrer et al., 2008) identified six main issues, as follows: (1) corporate strategy and financial models; (2) the resourcebased view of the firm; (3) industry and competition; (4) growth and market entry; (5) strategy process and top management; and, (6) strategy and its environment. Furrer et al. (2008) also identified a move in the direction of closer integration of the corporate and competitive (or business) levels of strategy that goes beyond the traditional hierarchical relationship between the corporate centre and the business unit. Corporate strategy is concerned with domain selection: what portfolio of businesses we are or should be in. Business strategy is concerned with how the business unit should compete in its respective market segments. In the analysis of the frequency of 26 keywords the keyword 'performance' ranked first, 'corporate' eighth, 'financial' tenth and 'innovation' twelfth. In an appendix, Furrer et al. (2008) provided a description of the content of each of the 26 keywords. Almost every keyword has a strong financial and management accounting dimension that is not evident in any review of the evolution of SM. In marketing terms, it seems that there 
are 25 important segments that are not being addressed by management accounting. Of course, marketers understand the importance of labeling, and the lack of agreement on a term for management accounting techniques used to support SM (see section 3, below) can certainly cause 'brand identity' problems (LangfieldSmith, 2008).

Cummings and Daellenbach (2009) undertook a similar study, a content analysis of 2,366 articles published in Long Range Planning in the period 1969-2006. The two studies differ in that the Long Range Planning articles reflect a more European perspective than do the four American journals in Furrer et al. (2008) The major themes that are of constant concern in Long Range Planning are consistent with the main 'issues' that emerge from Furrer et al. (ibid., p. 11). They are questions of the corporate whole; organising or structuring organisational resources; how an organisation responds to, or manages, change with regard to environmental developments, such as technological advances; decisions that relate to the above; processes or practices that influence such decisions; and creative innovative developments in any of these domains (Cummings and Daellenbach, 2009, p. 255). These six themes provide, in the view of Cummings and Daellenbach, a good basic definition of the bases of SM. Significantly, both studies observed that key spheres of recent investigation were in knowledge and learning, relationships, networks and, to a lesser extent, culture. Together with other reviews of the SM literature, these findings also help to delineate the empirical and conceptual context of SMA (Markides, 1999; Nadler and Tushman, 1999; Bowman et al., 2002; Herrmann, 2005; Grant and Jordan, 2012).

\subsection{Theoretical pluralism of SM}


The issues of constant concern established by content analyses studies do not, however, distinguish the different views that exist on each issue. The field of SM is distinguished by a history of theoretical pluralism that borrows concepts from several disciplines. For example, Mintzberg and Lampel (1999), identified ten schools of strategy, relating to base disciplines, which were more or less dominant in the last thirty or forty years and still influence practice and teaching today. Similarly, the principles of each school will be more or less relevant to the design and use of a SMA system, depending on the specific context. While most of the schools, notably the Cognitive (Psychology), Learning (Psychology and Chaos Theory), Power (Political Science), Culture (Anthropology) and Environment (Biology), are mainly descriptive, the Design (Architecture), Planning (Systems Theory and Cybernetics) and Positioning (Economics and Military History) schools adopt a more prescriptive stance, and the Entrepreneurial (Economics) and Configuration (History) schools are both descriptive and prescriptive. The schools and their base disciplines are part of the theoretical infrastructure that underpins the operation in practice of multidisciplinary teams that include accounting and finance. There is some evidence of a trend towards greater cohesion among the disparate disciplines of SM.

\subsection{A centred eclectism}

There is an acknowledged need for greater integration of disciplines within the field of SM, to go beyond the narrowness of each school, to understand the whole SM process and not just some parts of it; 'we need to know how strategy formation ... really works. The goal is better practice, not greater theory' (Mintzberg and 
Lampel, 1999, p. 29). One of the emerging trends that Cummings and Daellenbach (2009, p. 256) identified is 'a centred eclectism' where a diversity of approaches will still prevail but will focus on core topics such as organisation, process and change. 'This may prevent SM's eclectism leading to a field about everything in general and nothing in particular' (ibid.).

\subsection{Four perspectives of SM}

Two questions that pervade much of both SM practice and theory relate to how strategy is formulated and who strategy is for. The questions are as relevant to SM today as at any time in the last 50 years. Views on these questions highlight different ontological assumptions and ideological positions that are embedded in disparate forms of capitalism and social systems; they can be broadly described by four perspectives: classical, evolutionist, processualist and, the most recent, systemic (Figure 1, below). The classical and evolutionist perspectives both regard the maximisation of shareholder wealth as the overarching objective of the organisation. A key difference is that the classical perspective prescribes a formal, rational, linear approach, whereas evolutionists view the environment as unpredictable, and focus on building options, and increasing both strategic and organisational 'clock speed' (Nadler and Tushman, 1999 , p. 49, p. 52) in order to respond quickly to emerging situations. Processualists share the evolutionists' perception of environmental volatility but are skeptical of market efficiency and are more stakeholder oriented. Systemic theorists take a contingency approach, claiming that both the content of strategy and the strategic process are embedded in a network of social relations that may involve an extended enterprise and wider community (Granovetter, 1985). The 
relevance of the matrix and the questions, who is strategy for and how is it formulated, are perhaps best considered in the context of the environments that are shaping the SM process in organisations in the $21^{\text {st }}$ century.

\section{Figure 1 about here}

\subsection{How is strategy formulated?}

'Strategies need not be deliberate - they can also emerge more or less.... Smart strategists know that they cannot always be smart enough to think through everything in advance' (Mintzberg, 1989, p.29, p. 31). The changes in practice and in the literature from financial planning in the 1950s, to long-range planning in the 1960s, then to strategic planning in the 1970 s and finally to SM in the 1980 s reflect, in large part, an increasingly dynamic, unpredictable external environment. The economic, social and political turbulence of the early years of the $21^{\text {st }}$ century exemplifies the uncertainties that are causing 'smart strategists' to rely more on developing an organisational capability to respond quickly to new information. The collapse of the dot.com bubble on 10 March 2000 provided the first indication that the investment bubbles that had built up during the 1990s could not be sustained. The terrorist attack on the World Trade Centre in New York on 11 September 2001 was followed by wars in Iraq and Afghanistan, which had a major impact on trade, risk perceptions, political tensions and the economies of NATO countries, especially the USA and UK. The failure on 15 September 2008 of Lehman Brothers and the credit rating downgrade of American International Group on 16 September 2008, together, triggered a financial crisis that still has not been resolved. The on-going 
debt crises in European Union countries and political upheaval in several states in the Middle East have further deepened economic uncertainty.

The increasing frequency of seismic changes in the external environment and the uncertainties that they generate is one reason why an organisation's ability to respond quickly to possible new threats and opportunities has become a prerequisite to survival. Environmental volatility is a reason why many management commentators now argue that

'you have to give up the pretence that you can predict the future. This is about managing more dynamically....If old style (deliberate) strategy formulation is not exactly dead, then it is hardly in the best of health' (Stern, 2009, p. 14).

Nevertheless, there is a need for both deliberate and emergent strategies even if the deliberate strategy is more in the nature of broad purpose (Bartlett and Ghoshal, 1994, p. 79; Eisenhardt and Sull, 2001, p. 107); 'pushed to the limit, neither approach (deliberate nor emergent) makes much sense' (Mintzberg, 1989, p. 32). The waves of change in the $21^{\text {st }}$ century, the further intensification of competition and abbreviated technology, product and industry life cycles suggest that strategy formulation in most organisations may be moving along the deliberate-emergent continuum in the direction of the emergent end-point (Courtney et al., 1997; Courtney, 2009).

\subsection{Strategy-as-practice}


Although the concept of emergent strategy is a simple one and quite a lot is known about the analytics of deliberate strategy, relatively little is known about the processes and practices by which strategies are actually formulated and implemented. A rapidly growing body of 'strategy-as-practice' researchers are currently focusing more attention on the activities and discourse of strategy formulation, that is, on strategising rather than on strategy (Whittington, 2003; Chua, 2007; Johnson et al., 2007; Chapman and Chua, 2007; Jarzabkowski and Spee, 2009).

Six sets of questions for empirical research on strategising and organising were articulated by Whittington (2003, p. 119-21) namely:

'1. How and where is strategizing and organizing work actually done?

2. Who does the formal work of strategizing and organizing and how do they get to do it?

3. What are the skills required for strategizing and organizing and how are they acquired?

4. What are the common tools and techniques of strategizing and organizing and how are these used in practice?

5. How is the work of strategizing organized itself?

6. How are the products of strategizing communicated and consumed?'

Obtaining reliable empirical evidence to answer these questions is clearly a formidable challenge to researchers. Whittington (2003) suggests that the strategyas-practice approach to strategising and organising should start with a focus on formal processes because of: 
'... the relative ease with which we can trace it (the formal work) empirically .... While the instinct of the practice perspective is ethnographic, the formal work of strategic and organizational design offers units of analyses that are small and defined enough to allow for comparative case and even quantitative analyses' (p. 119).

The six questions are intended to establish what strategists' and organisers' jobs really are as a prelude to moving in a normative direction. This understanding requires 'developing stronger, practice-based links between micro- and macrophenomena' (Jarzabkowski and Spee, 2009, p. 90). MA can act as a powerful integrating vernacular in the process of linking macro-level strategic goals with

micro-level, operational targets (Nixon et al., 2011). The effectiveness of a combination of management accounting techniques, or SMA systems, depends very much on how it is used and on the extent to which it is a part of the organisational process that manages the formal, semi-formal and informal information and communication systems (section 4, below). This is the significance of strategy-aspractice research.

\subsection{Who is strategy for?}

Whether companies should seek only to maximise shareholder wealth or should endeavour to meet the often conflicting needs of stakeholders is a longstanding, controversial issue in the SM literature (Bowen, 1953; Davis, 1960; Frederick, 1960; Ackerman, 1973; Ansoff, 1982; Litz, 1996; Hillman and Klein, 2001; Smith, 2003; Friedman and Miles, 2006; Barton, 2011; Porter and Kramer, 2011). The word, 
'shareholder', is closely associated with the maximise shareholder wealth concept, short-termism and management practices that have negative connotations. Conversely, the stakeholder concept has come to be used in a variety of contexts; there is a 'profusion (of stakeholder definitions) and some confusion' (Friedman and Miles, 2006, p. 3). Whittington (2001) uses the words, 'profit-maximising' and 'plural', which avoid to an extent the shareholder-stakeholder tensions and the difficulties of managing, for example, the triple bottom line of profit, people and planet (Savitz and Weber, 2006).

The shareholder-stakeholder orientation of an organisation is important because it affects both the content of strategy and the SM process. Financial institutions and shareholders clearly influence a company's position on the shareholder-stakeholder continuum (Marsh, 1990; Plender, 2003; Haldane and Davies, 2011; Sappideen, 2011). However, the corporate scandals that have persisted since Enron in 2001, and the recent financial crises and corporate governance practices have generated an anti-business sentiment, which goes well beyond fringe minority groups. Ethics, equity and environmental sustainability have become mainstream social and political issues that may change the shareholder-stakeholder balance (Grant and Jordan, 2012). Michael Porter, a traditional maximisation of shareholder wealth advocate, wrote recently in a co-authored article that 'Capitalism is under siege ... trust in business has fallen to new lows.... Shared value could reshape capitalism and its relationship to society' (Porter and Kramer, 2011, p. 181). Movement on the shareholder-stakeholder continuum seems to be driven, in large part, by the choice that business leaders now face: 'they can reform the system, or watch as the government exerts control' (Barton, 2011, p. 85). A further influence is the fact that 
'[l] oss of social legitimacy may be as big a threat to the survival of commercial and investment banks as their weak balance sheets' (Grant and Jordan, 2012, p. 463).

One implication of movement within the four perspectives (classical, evolutionist, processualist and systemic), or of a narrowing of the differences among them, is that the concepts and tools of each perspective need to be modified both technically and operationally. For example, the Balanced Scorecard and the Tableau de Bord are grounded in different social and economic systems (Bourguignon et al., 2004), and although they may not converge, the distance between them is likely to be changing.

\subsection{Institutionalising change}

The history of the evolution of SM provides a useful, 'wide-angle' lens through which to view the theory and practice of SMA. One thing is clear: the velocity and interacting nature of changes in the external environment are transforming the SM tools and concepts of practitioners, consultants and researchers (see Table 1). Over a decade ago Nadler and Tushman (1999) observed that '[h]istorically, the purpose of organizational structure was to institutionalize stability; in the organization of the

future, the goal of design will be to institutionalize change' (p. 49). More recently Gary Hamel (2012) stated that 'we are going to see a greater revolution in how companies are run and managed over the next decade than we've seen over the last 100 years' (Hill, 2012, p. 16). The strategic and organisational imperatives of these environments are the 'shifting landscape' (Jacobides, 2010, p. 77) of SM and SMA.

\section{SMA literature}


Several reviews of the SMA literature have been conducted in the last few years (Nyamori et al., 2001; Roslender and Hart, 2003; Chenhall, 2005; Bhimani and Langfield-Smith, 2007; Langfield-Smith, 2008; Tillman and Goddard, 2008; Bhimani and Bromwich, 2010). The purpose here is rather to evaluate the scope of the SMA literature in the context of the evolution of SM to identify the issues, trends and perspectives of SM that are not currently being addressed by SMA research.

\subsection{The concept of SMA}

Simmonds' concept of SMA in 1981 was based more on Porter's frameworks than on the second-era resource-based view of SM. Simmonds defined SMA as 'the provision and analysis of management accounting data about a business and its competitors, for use in developing and monitoring business strategy' (Simmonds, 1981 , p. 26). The stream of research that Simmonds' definition catalysed focused more on the cost management needed to support low price competitive strategies (Shank, 1989; Bromwich, 1990; Cooper, 1995, 1996a, 1996b) than on the research, development, design and innovation required to earn a price premium through product differentiation (Porter, 1980).

It is, perhaps, hardly surprising that '[t]here is no agreed definition of SMA in the literature' (Langfield-Smith 2008, p. 205). In part, the disparate definitions of SMA may be attributed to the difficulty of defining precisely a concept as dynamic and multi-faceted as strategy: 'In an age when nothing is constant, strategy should be defined by narrative - plots, subplots and characters - rather than by maps, graphs, and numbers' (Jacobides, 2010, p. 77). This latter view is clearly different from the strategy- map approach of Kaplan and Norton (2001b, 2004) but it is consistent with 
the more emergent nature of strategy and the strategy-as-practice approach (see section 2.8, above). Although there is a broad consensus that SM is about planning, implementation and control, each of the ten schools and four perspectives of SM (see sections 2.4 and 2.6, above) interpret these three elements quite differently; for example, how, by whom and for whom they should be conducted. The many dimensions of SM do not readily support a broadly agreed definition of SMA.

Traditionally, management accounting has been conceived of as supporting the financial decision-making process in organisations (Ward, 1992; Feeney and Pierce, 2007; Drury, 2008; Bhimani and Bromwich, 2010). The three roles of the financial function (management accounting, financial accounting and treasury) are closely related and have become more, rather than less, integrated. One reason for this fusion of the financial roles is the trend away from the diversified conglomerates of the 1960 s and 1970 s to technology- and industry-focused groups.

The relatively high frequency of the keywords, 'financial' and 'finance', in the longitudinal, content analyses of the SM literature by Furrer et al. (2008) and Cummings and Daellenbach (2009) also suggests that the treasury, management accounting and financial accounting roles have been subsumed within the more generic 'finance' term in the SM literature.

Establishing a clear identity and boundaries for SMA is complicated further by the loosely-linked nature of the management accounting techniques associated with SM. For example, a survey by Guilding et al. (2000), conducted in New Zealand, the UK and the USA used 12 techniques. Another survey of Italian manufacturing firms by Cinquini and Tenucci (2007), referred to 14 techniques in their questionnaire. Cadez and Guilding (2008) used 16 'strategically oriented accounting techniques' (p. 838) in their survey of Slovenian companies. The 16 techniques fall into five categories: (1) 
costing, (2) planning, control and performance measurement, (3) strategic decision making, (4) competitor accounting and (5) customer accounting. The categories are reinforcing in so far as they relate to competitors, customers and attainment of competitive advantage. Investment appraisal seems to have been dropped from these recent lists of SMA techniques (Cadez and Guilding, 2008).

The term, SMA, was not adopted in the USA, notwithstanding the preoccupation of researchers and consultants with SM since 1977 and the claims of high-profile academics, such as Johnson and Kaplan (1987) that 'management accounting systems are not providing useful timely information for the process control, product costing, and performance evaluation activities of managers' (p. xix). Instead a 'strategic cost management' concept emerged that sought to adapt 'the traditional body of knowledge called cost analysis to the rapidly developing body of knowledge on strategy formulation and implementation' (Shank and Govindarajan, 1993, p. 1). Although the strategic cost management framework was comprehensive and conceptually robust, it was not widely adopted; and, by 2006 Shank expressed disappointment that strategic cost management was mostly 'evolving outside the purview of the accounting profession' (Shank, 2006, p. 359).

\subsection{The Links between the SM and SMA literatures}

Most of the published empirical research over the past 30 years has consisted of questionnaire surveys that sought to establish the extent to which specific SMA techniques were adopted (Langfield-Smith, 2008). However, the limitations of surveys and the relative dearth of case studies mean that very little is known about how the SMA techniques are used, by whom and for whom (see section 2.8, above). 
For example, in order to manage a set of SMA techniques effectively, it is necessary to understand how they relate to other techniques in the formal information system.

Furthermore, the techniques may be used simultaneously and/or sequentially, depending on the nature of the decision, context or phase in the SM process. The formal information system itself is embedded in the whole system that includes the structure, management style, including leadership, the informal system and culture (Granovetter, 1985; Roberts, 2004). Empirical evidence from the SM literature supports 'complementary theory predictions that it is whole system change that delivers the significant pay-offs .... Piecemeal initiatives typically cost more than they are worth' (Whittington et al., 1999, p. 3). The SMA literature has mostly not addressed the constant themes over the last three decades in the SM literature change, organisational resources, innovation and the corporate whole (see section 2.3, above).

The topical shareholder-stakeholder issue has also been largely neglected in the SMA literature. The formal, structured, linear approach that pervades the SMA literature is based on two classical perspective assumptions, namely that the environment is relatively stable and predictable and that the overarching objective of the organisation is maximising shareholder wealth. In effect, the SMA literature takes relatively little account of the evolutionist, processualist and systemic perspectives. Yet the assumptions underlying these latter three perspectives may be more relevant to SM in the context of a particular organisation than those of the classical perspective. Of course, the perspectives are not mutually exclusive; deliberate and emergent strategies co-exist, and even within the same organisation the shareholder-stakeholder orientation may vary in relation to sub-groups, depending in 
large part on the relationship and bargaining power of groups with the organisation (Pfeffer and Salancik, 1978; Kennedy, 2000).

The classical perspective orientation of SMA is long-standing. Most of the papers in the previous special issue in Management Accounting Research on SMA focused 'quite narrowly on formal aspects of SMA analysis ... [but] there is a far broader dimension of SMA to pursue' (Tomkins and Carr, 1996, p. 272). However, over a decade later there is still little evidence of the wider recognition of these informal roles.

This gap between the trends, themes and process of SM and the SMA literature has been the subject of recurring criticism. Nyamori et al. pointed out that SMA did not question 'what strategy is, how it is formed, how it comes to change, and how strategic change constitutes and is constituted by accounting' (2001, p. 63). The SMA literature is based in large part on a narrow, first-era, view of the SM literature (see section 2.1, above).

The Bhimani and Langfield-Smith study (2007) which included comprehensive reviews of both the SM and SMA literatures, concluded that '[t]he scholarly conceptions of strategic management that view strategy development and implementation as emergent, unstructured and in continual flux have only selectively been integrated within the SMA literature' (p. 9).

A recent case study, however, suggests that the SMA literature may not correctly reflect SMA practice and the role of management accounting in the SM process of organisations. The case study by Tillman and Goddard (2008) of a German multi-national company may also reflect the important influence of national culture on the design and use of MA in the process of developing and implementing strategy (Granovetter, 1985; Tomkins and Carr, 1996; Chow et al., 1999; Lindsay, 
2000). The case describes in considerable detail how SMA is perceived and used by participants in a dynamic SM process, which is very much a 'sense-making' activity that entails 'structuring and harmonizing', 'bridging and contextualizing' and 'compromising and balancing' (Tillman and Goddard, 2008, p. 80). The 'sensemaking' role of SMA is completely consistent with a trend that Cummings and Daellenbach (2009) predicted would shape SM in the next decade, namely, the trend for 'less focus on command and control, more on influencing, steering, nudging, connecting interests and internal marketing' (p. 257). Johnson (1992) anticipated this trend in his book, 'Relevance Regained: From Top-Down Control to Bottom-Up Empowerment'.

\subsection{Some limitations of the SMA literature}

One limitation of SMA relates to its definition and another to the nature of the related empirical evidence. The various definitions of SMA mostly share a common focus on competitive and marketing strategies (Langfield-Smith, 2008) and the management accounting techniques that might support them (Carr et al., 1991; Shank and Govindarajan, 1992; Carr and Tomkins, 1996; Kaplan and Cooper, 1998; Guilding et al., 2000; Cadez and Guilding, 2008). A problem with this focus is that the context and nature of competition has changed dramatically in the last decade. Porter's competitive strategies $(1980,1985)$ have not stood 'the test of time' (see section 1, above). Even by 1993 it was evident that differentiation at low cost had become an entirely feasible strategy (Lorenz, 1993); yesterday's bases of 
competitive advantage had become today's price of entry (McNair and Liebfried, 1992). The scope and content of the marketing mix has become much broader and more complex. One reason for the demise of strategic cost management in the 1990s (Cooper, 1995, 1996a, 1996b; Shank, 2006; Langfield-Smith, 2008) may be that, increasingly, firms need to compete on several bases simultaneously, for example, on product features, price, quality, functionality, service and image. One implication of the need to compete on several bases simultaneously is that '[c]ost management is not an isolated art, or one that should be confined to the accountants' floor. It should involve the whole business' (Hopwood, 2003, p. 11). The context and nature of the cost, value and other financial calculations, required to support sustainable competitive and marketing strategies, are changing in ways which suggest that the extant SMA literature may be lagging behind practice, as discussed in Section 4 (below).

The external and long-term orientation criteria for SMA techniques, identified by Guilding et al. (2000) are certainly necessary but they risk eclipsing the need for internal organisational capabilities to support external competitive bases. It is also the case that the speed of change in the external environment is substantially shortening industry, technology and product lifecycles. In some sectors executives now talk about strategic cycles in terms of 'web years', signifying compressed timeframes of three months rather than 12 (Nadler and Tushman, 1999, p. 51).

A second limitation is that with just a few notable exceptions (for example, Rickwood et al., 1990; Collier and Gregory, 1995; Lord, 1996; Dixon, 1998; Seal, 2001, 2010; Bhimani and Langfield-Smith, 2007; Tillmann and Goddard, 2008; Jorgensen and Messner, 2010), the SMA research studies conducted mostly 'throw 
little light on how SMA practices are implemented and used in practice and provide no theoretical explanation of such practices' (Tillmann and Goddard, 2008, p. 81). The narrow, technical focus of SMA addresses just a small part of the SM literature and neglects the interaction among many organisational components that the SM process, whether deliberate and/or emergent, entails. '[T]he interplay between strategic action and accounting practice needs to be understood in terms that are reflective of the complexity of organizational uncertainties and social processes which condition their relationships' (Bhimani and Langfield-Smith, 2007, p. 8).

\section{The SMA literature and practice}

There is a dearth of evidence 'on how SMA-inspired techniques and processes diffuse into general practice within organizations' (Langfield-Smith, 2008, p. 204).

\subsection{SMA in the context of current product development process}

Two recent case studies, that address this knowledge-gap, focus on management accounting in the context of knowledge-intensive, complex and highly uncertain new product design and development (NPD\&D) processes. The NPD\&D activity of both companies, referred to here for convenience as Company A (Jorgensen and Messner, 2010) and Company B (Nixon et al., 2011), is crucial to their competitiveness and financial performance. Accounting and financial numbers pervade the entire NPD\&D process of both A and B, especially at 'stage gates'. In

Company B, a UK subsidiary of a German multi-national motor manufacturer, the NPD\&D process has 14 stages and five phases. The term 'strategic management 
accounting' is not part of the communication lexicon of either company, notwithstanding the extensive use of financial criteria and accounting to support resource allocation decisions among product portfolios, to manage constraints, evaluate irreversible decisions, options and trade-offs, and to assist compromise and attainment of balance among competing objectives and participants, including customers, in the NPD\&D process.

The concepts of accountability and empowerment, that are essential to strategising and organising, are very clearly understood in both A and B; in Company B they are explicit components of the business model that also includes customer orientation, trust and teamwork. Most of the SMA techniques, identified by Cadez and Guilding (2008) are part of the information system operated by B; in addition, several investment appraisal techniques are used to evaluate all NPD\&D expenditures and related risks. Many of the SMA techniques are embedded in longestablished design and development systems, such as Quality Function Deployment, Failure Mode Effect Analysis, Design for Manufacture and Assembly and the new generation of more integrated NPD\&D systems, such as Product Data and Product Lifecycle Management systems.

The management of NPD\&D project teams in Company $A$ is different from that in B, and highlights the need for a contingency approach (Cadez and Guilding, 2008). Company A employs a separate team for each of the three phases in its NPD\&D process. In B the same NPD\&D project team is responsible for all five phases and 14 stages of the process. The primary reason for this latter arrangement is that in Company B's experience it enhances communication, both formal and informal, and helps to avoid expensive delays and errors. The one-team-only arrangement for each project also enhances the sense of identity and ownership that individuals have 
with particular vehicle models. Fast strategic response and time-to-market are critical success factors for $B$ and are a major influence on the design and operation of the company's business model.

Another difference is that in Company A there is 'a general understanding of the need to be profitable' (Jorgensen and Messner, 2010, p. 202), whereas in B profit requirements are explicit from the outset. Careful estimates of return on investment and risk evaluation are an essential part of the business case that must be made for each project in B. Accounting is the common language that links all participants in the NPD\&D process of Company B, both horizontally over the one to three years of a major project and vertically in each stage of a process. For every project there are four management levels - the Product Strategy Committees of B and its parent company, the project manager and module leaders. However, accounting in $B$ is simply one part of a tightly integrated set of organisational components that are used to manage communication. These complementary, reinforcing components are:

(1) a relatively horizontal, team-based structure;

(2) a comprehensive, very accessible formal information system;

(3) an iterative, top-down, bottom-up management style;

(4) greater emphasis on planning and prospective-type information than on control and retrospective information;

(5) a powerful informal information system that supports the formal system, especially through the management style and team-based organisational structure; (6) an open, trusting culture, grounded in a century of engineering excellence and an acute understanding of the need for both sound financial management and constant innovation in order to survive. 
These six components and the interactions among them are managed very much as a single system in order to maintain the internal consistency necessary to support B's competitive and marketing strategies. This organisational embeddedness of SMA in B contrasts sharply with SMA in the GEC/Marconi case study, reported by Seal (2010). Seal found that the SMA system has no practical impact and that 'there is little evidence of it as an organizational sensemaking device, as a managerial discourse and certainly no evidence of institutionalization' (p. 107). These two cases, $A$ and $B$, exemplify the crucial importance of institutionalising the SMA system.

\subsection{A worrying lack of knowledge}

The intensification of competition that has increased the importance of the early SMA focus on competitive and marketing strategies has also increased the need for a steady stream of new and improved products and services to support competitiveness. Yet notwithstanding the broad consensus on the importance of the new product design and development (NPD\&D) activity and innovation to sustained performance of organisations (Davila, 2005; Chesbrough, 2006, 2011; Nagji and Tuff, 2012; Thomke and Reinertsen, 2012), 'there remains a worrying lack of knowledge of what it actually means to practice accounting in new product development' (Jorgensen and Messner, 2010, p. 186). Although the two case studies, A and B, referred to above, are clearly no basis for empirical generalisation, several trends in the management and technology of the NPD\&D process in the last 20 years suggest that accounting/financial management plays a key role in resource allocation decisions and in the operational management of the NPD\&D activity. In particular, the costs, risks and complexity of NPD\&D are increasing as companies 
are compelled to move from product-by-product development to developing platforms for two or three generations of several families. Within B, for example, a platform has a life expectancy of 14 years and normally supports at least two brands for two generations. The trend toward more service-based 'solutions' changes fundamentally the nature of risks and revenue streams. Faced with these formidable financial management challenges, the apparent low uptake of SMA seems paradoxical. Much more evidence is required as well as a broader interpretation of the term SMA.

\section{SMA and the related literature}

The theoretical pluralism, diversity of perspectives and the trend toward a 'centred eclectism' (see sections 2.4 and 2.5, above), that are evident in the SM literature, are not mirrored in the SMA literature. Similarly, trends in practice, such as continuous innovation (Ries, 2011), more intensive cross-functional collaboration in multidisciplinary teams (Beyerlein et al., 2000; Th et al., 2002; Ahmed et al., 2008), greater integration of systems (Brown et al., 2011), flatter, more horizontal organisational structures (Chenhall, 2008), and collaborative alliances (Lawrence et al., 2005), are not well addressed in the SMA literature. Cognate strategy-oriented literatures, such as strategic control (Atkinson et al., 2001; Chapman, 2005), management control (Widener, 2004; Berry et al., 2005; Henri, 2006; Macintosh and Quattrone, 2010; Merchant and Van der Stede, 2012), knowledge management (Keen and Scott Morton, 1978; Grant, 1996; Kazanjian et al., 2002; Bhimani and Roberts, 2004), and performance measurement (Kaplan and Norton, 1992, 1996; 2001a; 2001b; 2006; 2008; Olve et al., 1999; Ittner and Larcker, 2005; Smith, 2005), 
are more pluralistic and address more directly than does SMA developments in both competitive and marketing strategies and in the resource-based view of competitive advantage. This broader, more integrative approach of SMA-related literatures means that: '[w]e can now say very sensible things about the relationship of accounting to organizational strategies, structures and designs' (Hopwood, 2005, p. $585)$.

The management control and performance measurement literatures have developed rapidly since publication of the books by Simons (1995) 'Levers of Control', and Kaplan and Norton (1996) 'The Balanced Scorecard'. The preoccupation with performance and its management has helped the development of strong conceptual and practice links between the performance measurement and management control literatures (Simons, 2000; Hartmann, 2000; Otley, 2003; Verweire and Van den Berghe, 2004; Henri, 2006; Chenhall and Langfield-Smith, 2007; Cokins, 2009). The fusion of performance measurement and management control and 'the transition (of management control) into performance management' (Otley, 2003, p. 309) are consistent with the trend from functional to multidisciplinary, team-based structures (Mullern, 2000; Bhimani and Bromwich, 2010). The convergence of management control, performance measurement and performance management is also consistent with the pervasive principle of complementarity theory (Milgrom and Roberts, 1995) that emphasises the importance of alignment and the need for an array of mutually reinforcing activities to support strategy. However, the links between the SMA literature and related literatures, such as performance measurement, management control and knowledge management, in particular, are weak. Although Roslender and Hart (2002) included Simons' model in their framework for integrating management accounting and 
marketing, the various sets of management accounting techniques used for most of the published empirical SMA research in the last 25 years (Langfield-Smith, 2008) have been only tenuously linked with the management control literature.

The SMA literature also takes virtually no account of the emerging knowledge component of the resource-based approach to competitive advantage (Grant, 1996). Yet the analysis and aggregation of data that SMA techniques facilitate can greatly enhance the knowledge management, communication co-ordination that are essential for effective SM. The gap that exists between SMA and the SM literature (see section 3.2, above) is easier to understand than the gap between SMA and more closely related literatures.

\section{Discussion and conclusion}

\subsection{The paradox of SMA}

The SM academic and practice-oriented literatures have developed rapidly in parallel with a related SM consultancy business since the launch of the Strategic Management Journal in 1979. In sharp contrast to strategic management, SMA has 'remained a collection of academic texts and has had a negligible impact on managerial discourse and practice' (Seal, 2010, p. 95).

There is usually neither a simple explanation nor an easy solution to an apparent contradiction. For example, 'companies ... will need more management accounting information but fewer management accountants' (Cooper, 1996a, p. 20). Cooper suggested two related reasons to explain his prediction; the pressure on organisations to reduce costs, while simultaneously meeting the product-service 
quality and functionality that customers demand, and using distributed systems to decentralise the management accounting process. Cost management was becoming everyone's responsibility. As Hopwood (2003) observed, cost management is also a strategic issue that 'has the potential to change the whole business' ( $p .11)$. Both the pressures on costs and the diffusion of very accessible cost and management accounting techniques have continued to increase since Cooper's warning. These trends are one reason why the demand for SMA techniques might also be expected to have increased. The burgeoning SM literature and consultancy business are another reason.

A definitive explanation is clearly preferable to a tentative one. However, for several reasons circumspection is required when interpreting the survey evidence that SMA techniques have not been adopted widely. These reasons include the lack of consensus on what SMA is (see section 3.1, above), the diffusion of management accounting techniques within organisations and their extended networks and the low recognition of the SMA 'brand name' (Bhimani and Bromwich, 2010, p. 48). The small number of case studies that have been conducted confirm the use of management accounting techniques to support SM within these organisations. The case study evidence also highlights the low recognition of the term SMA even when management accounting concepts and techniques are a part of the whole system of $\mathrm{SM}$ in these organisations (see section 4, above). '[D]isagreement concerning definitions is not of major importance; much more important is that SMA is employed by firms' (ibid., p. 49).

The gap between the SMA literature and practice is exacerbated by the gaps between SMA and the SM literature (see section 3.2) and the cognate literatures of performance measurement, management control and knowledge management (see 
section 5.). This lack of integration and identity may begin to explain the apparent low adoption of SMA techniques.

\subsection{Areas for future research}

The strategic and organisational imperatives of a dynamic external environment mean that the context of research areas is constantly changing. The above analysis points to four related areas of future research, namely, the gaps between the SMA literature and (1) the SM literature (see section 3.2, above); (2) practice (see section 4, above); (3) cognate strategic-oriented literatures (see section 5, above); and (4) the lack of consistency, cohesion and coherence among techniques attributed to SMA (see section 3.1, above).

\subsubsection{The links between the SMA and SM literatures}

The second era of SM that began to evolve in the late 1970s has been addressed only in a very limited way in the extant SMA literature (see sections 3.2 and 3.3, above). This gap suggests that SMA researchers could usefully begin to explore the management accounting concepts and techniques relevant to the issues and perspectives of the SM literature (see sections 2.3-2.9, above). Anderson (2007) pointed to research in other disciplines, such as marketing, operations management, business strategy, finance and economics, that had 'already laid the groundwork for understanding strategic cost management' (p. 498). Although these other disciplines 'tend to present a circumscribed view of cost management in a narrow portion of the value chain' (ibid.), Anderson argues that 'the new challenge for cost management 
research is to engage with diverse research streams ... and to integrate what has been learned in other disciplines with management accounting theory' (ibid.). From a SMA perspective the ideal approach would be for multi-disciplinary teams, working in an iterative way to and from the SM and SMA literatures, to conduct in-depth case studies. SMA research needs to be part of the SM research trend toward 'a centred eclectism' (see section 2.5, above). There can be little doubt that, in the context of SMA research especially, 'leveraging diversity can only bring richer knowledge' (Davila and Oyon, 2008, p. 887).

\subsubsection{The links between SMA literature and practice}

'[l]nfluencing practice is an important objective of management accounting' (ibid., p. 888). However, the relationship between SMA research and practice is very much a reciprocal one.

The SMA system of Company B (see section 4, above) is an integral part of the organisational design that supports SM of the three processes (customer relations and marketing, NPD\&D and support ${ }^{1}$ ) of the company's business model. By studying SMA within the organisational context it is possible to begin to understand the interaction among all the dimensions that can influence SM, of which SMA needs to

\footnotetext{
${ }^{1}$ The support process of company B includes the following sections: Finance, Legal, Information Systems, Strategy and Target Setting, Plant and Facilities, and People. The SMA system is managed by a multi-disciplinary team in Strategy and Target Setting; the head of this section has a dual responsibility to the CEO and CFO.
} 
take account - for example, internal and external dimensions, strategic and operational, formal and informal, explicit and tacit, technical and social/behavioural. 'Enterprise-based longitudinal studies' (Bhimani and Langfield-Smith, 2007, p. 25) also allow researchers to locate the SMA system, to establish how it is used, by whom, for what purpose and how the various analyses and measures are synthesised. 'Organisational nuances' also become more apparent (ibid.). For example, in Company B the formal information system, including the SMA, influences indirectly the informal system through the formal team structure and a topdown, bottom-up iterative management style. The formal information system is actually managed as a dynamic continuum of structured, semi-structured and unstructured (informal) information.

Case studies also provide an opportunity to assess who owns the SMA system (Langfield-Smith, 2008). Within Company B the system is compiled and managed by the 'Strategy and Target Setting' department. However, ownership belongs to the teams responsible for providing much of the data; this ownership arrangement encourages team participants to share information and to use the system. Economists and knowledge management theorists are familiar with the 'appropriability' problem, namely how to ensure that the owner of a resource, such as research and development knowledge or a design receives a return equal to the value created (Teece, 1987).

"Does it matter if the SMA developments are not managed or "owned" by the accounting function?'(Langfield-Smith, 2008, p. 223). A study that focused on the strategic information needs of 12 senior managers in six leading Dutch organisations concluded that 'strategic accountants can bridge the gap between traditional 
accounting and SM and provide strategic managers with the type of information they need to make informed, timely decisions' (Brouthers and Roozen, 1999, p. 321). Although Anderson (2007) is 'ambivalent about the need for specially trained practitioners, who work in accounting departments and employ a narrow set of management accounting tools to analyze data' (p. 498), she nevertheless believes 'that management accounting has a natural role in both the strategic decisions that define the cost structure for the long term as well as the effective execution of these strategies in the short term' (ibid.).

Of course, management accounting is conducted by many disparate disciplines (Lord, 1996; Dixon, 1998). Bhimani and Bromwich (2010) point out that '[t]here is a danger that the investment and cost aspects of strategy may be considered secondary in the absence of accountants being involved in strategy and able to apply SMA techniques' (p. 52). From the perspective of the multi-disciplinary teams that accountants increasingly operate in, the question of ownership of the SMA activity seems redundant and potentially dysfunctional; the SMA belongs to the team, although accountants are likely to have a competitive advantage in compiling and using financial numbers, balancing and negotiating financial targets among other disciplines, analysing variances and ensuring consistent application of financial criteria in decisions. A further advantage that accountants enjoy is that their financial assessments of marketing, operations, or NPD\&D decisions are likely to be perceived by senior management as relatively more objective than those of the discipline managers directly concerned. From this perspective, collaboration and communication across disciplines are much more important than the question of SMA ownership. 


\subsubsection{The links between the SMA literature and related literatures}

The many techniques of SMA, including the executional and structural cost management tools of strategic cost management, can provide depth to, and help to integrate, the perspectives of performance measurement and the controls of management control. The performance measurement and management control literatures, which have already moved closer (Simons, 2000), can, in turn, help to provide a basis for integrating the fragmented developments of both SMA and strategic cost management into 'a unified body of knowledge' (Anderson, 2007, p. 498). The techniques of SMA can also be developed by, and, in turn, enhance, concepts of knowledge management relating to, for instance, uncertainty and risk management, innovation, knowledge transfer, communication and co-ordination. Future research could explore, for example, how the potential synergies among SMA, performance measurement, management control and knowledge management can be better exploited.

\subsubsection{Towards an integrated framework}

The evidence of this paper suggests that a unified body of knowledge for SMA would have four 'building blocks': the SM literature, practice, related strategyoriented literatures and an integrated set of management accounting techniques. Future research could endeavour to understand and develop the interactions and complementarities among the four blocks and also among the many management 
accounting techniques that comprise SMA. Anderson (2007) observed that, in the context of strategic cost management, 'management accounting research has tended to focus on executional (operational) cost management and on the product (manufacturing) portion of the value chain' (p. 497). She suggested that researchers could attempt to extend the scope of cost management and to make it more strategic by also focusing on 'structural cost management' that 'employs tools of organizational design, product design and process design to build a cost structure that is coherent with strategy' (ibid., p. 481). Both structural and executional cost management techniques are embedded in the NPD\&D process of Company B (see section 4 , above). Part of the paradox of SMA is that there is so little research in an area with so much scope for potentially rewarding research. One possible explanation may be that the scope and theoretical pluralism of SM make it imperative for SMA researchers to cross conceptual and discipline boundaries. 'However, as management accounting researchers we can be criticized for working too much within our silos' (Davila and Oyon, 2008, p. 887). Future research that aims to strengthen the links among SMA, SM, practice, cognate areas and SMA techniques (see sections 6.2.1 -6.2.4) requires interdisciplinary research. There is a great opportunity and also an acute need to 'leverage (the) diversity' through 'crossparadigm collaboration' (ibid.). 